Voix et Images

voixetimages

\title{
Comédie, la mal-aimée
}

\section{LUCIE ROBERT}

Volume 42, numéro 2 (125), hiver 2017

URI : https://id.erudit.org/iderudit/1039926ar

DOI : https://doi.org/10.7202/1039926ar

Aller au sommaire du numéro

\section{Éditeur(s)}

Université du Québec à Montréal

\section{ISSN}

0318-9201 (imprimé)

1705-933X (numérique)

Découvrir la revue

Citer ce compte rendu

ROBERT, L. (2017). Compte rendu de [Comédie, la mal-aimée]. Voix et Images, 42(2), 179-184. https://doi.org/10.7202/1039926ar d'utilisation que vous pouvez consulter en ligne.

https://apropos.erudit.org/fr/usagers/politique-dutilisation/ 


\author{
D R A M A T U R G I E \\ Comédie, la mal-aimée \\ $+++$
}

LUCIE ROBERT

Université du Québec à Montréal

"On discute mal sur papier avec la comédie ${ }^{1}$ », observe Gilbert Turp, car son écriture est destinée moins à être lue qu'à passer par le corps ou la voix d'un acteur. L'abondance des textes comiques sur les scènes et sur les ondes ne devrait pas masquer le fait que faire rire suppose une écriture rigoureuse, et qu'il est difficile d'atteindre un comique véritable, dénué de toute facilité burlesque, pour traduire non pas une réalité, mais un point de vue inconvenant, irrévérencieux, moqueur ou satirique sur cette réalité. La comédie emprunte ainsi plusieurs modèles ou registres, soit qu'elle tende vers la comédie d'intrigue, qui n'est pas dénuée de sérieux voire de pathétique, ou, à l'opposé, qu'elle succombe à la tentation de la farce la plus grossière. Dans l'histoire, le genre n'a guère été défini autrement que par ce qu'il n'est pas, et les théoriciens comme les critiques n'y ont porté qu'une attention distraite, contrairement à la tragédie ou au drame qui, en leur temps, ont suscité de nombreuses réflexions. «Genre mineur, rappelle Marie-Claude Canova, [la comédie] reste implicitement au bas de la hiérarchie des genres dramatiques ${ }^{2}$.» Peu d'auteurs y ont vraiment excellé; encore moins nombreux sont ceux qui en ont tiré leur légitimité historique. On citera pour mémoire le maître incontesté du genre, Molière, bien sûr, qui a néanmoins dû en repenser la forme et la structure pour obtenir la reconnaissance de son siècle, mais aussi Dario Fo, récemment décédé, dont le prix Nobel avait fait sourciller quelques esprits bien-pensants: donner le Nobel à un clown ne fait pas sérieux, paraît-il. Et même s'il est vrai que «[l]a cause du rire est une de ces choses plus senties que connues ${ }^{3}$ ", du rire et du comique il sera question ici.

$+$

L'on sait que l'humour représente au Québec une industrie considérable: près de la moitié des spectacles présentés sur scène relève du numéro comique. À peu près rien

1 Gilbert Turp, La culture en soi. Essai, Montréal, Leméac, coll. «Thêâtre. Essais», 2006, p. 80.

2 Marie-Claude Canova, La comédie, Paris, Hachette, coll. «Contours littéraires», 1993, p. 3.

3 Adrien-Jean-Quentin Beuchot, "Avertissement", Voltaire, L'enfant prodigue, comédie en cinq actes, 1738 [1736], en ligne: https://fr. wikisource. org/wiki/L’Enfant_prodigue_(Voltaire) (page consultée le 8 février 2017). 
de cette activité ne rejoint le marché du livre. Ceux de ces spectacles qui sont destinés à durer sont plutôt enregistrés sur disque ou sur un support équivalent. Ne nous parviennent ainsi que de très rares exemples de la pratique d'un genre bref (le numéro), qui mise en général sur le comique de situation. Citons le recueil de sketches de Denis Bouchard et Rémy Girard, Tranches de vie ${ }^{4}$, version retravaillée d'un spectacle intitulé La farce de l'âge, créé au Théâtre du Rideau Vert en 1990. Que les auteurs aient mis autant de temps à livrer ces textes à la lecture laisse cependant songeur, d'autant plus que, dans ce domaine, l'effet de caricature se démode rapidement. Chacun des numéros s'inspire d'un genre musical particulier (jazz, ballade, punk), qui lui sert de sous-titre, et trace un portrait caustique de la famille, du moins de ce qu'elle est devenue au cours de ces années, s'appuyant en particulier sur le brouillage des genres ou des générations qu'engendrent la composition, la décomposition et la recomposition de l'unité familiale. Ainsi un jeune enfant reste confus devant les horaires de garde partagée que lui ont concoctés ses trois (ou quatre) familles; un jeune couple qui craint les maladies se donne rendez-vous à la pharmacie plutôt qu'au restaurant ou au cinéma; des adolescents prennent possession du domicile familial et relèguent leurs parents aux petites pièces. Dans l'histoire du comique, les relations familiales représentent en effet un sujet qui paraît ne jamais devoir s'épuiser. Force est de constater que ces numéros au rire un peu gras s'adressent à un vaste public d'une manière sans doute trop consensuelle, et que leur succès est dû en bonne partie au talent et à la notoriété des deux comédiens, écrivains improbables, qui livrent néanmoins leurs textes avec une certaine générosité.

Par comparaison avec la logique du spectacle comique à numéros, la comédie légère offre déjà une construction plus complexe. «La comédie m'a permis de dire ce que j'avais à dire en paix» (10), écrit Catherine Léger dans la présentation de $J^{\prime}$ ai perdu mon mari $^{5}$, créée au Studio Hydro-Québec du Quai des arts de Carleton-sur-Mer le 15 juillet 2014, dans une mise en scène de Philippe Lambert. La pièce renoue avec la structure rigide des trois actes, pour mettre en scène l'histoire d'Évelyne qui, un soir où il était tard, a parié son mari qu'elle pourrait chanter en karaoké toutes les chansons du livre du bar Capri. La suite de la pièce traite essentiellement des conséquences de ce désastreux pari. En effet, Mélissa, la serveuse, a hérité du mari en question alors qu'Évelyne, désormais privée de la BMW, reste aux prises avec William, son beau-fils un peu paumé. L'affaire se complique de ce que William est amoureux de Mélissa, qui elle-même découvre les aléas de la vie d'un couple asymétrique tant par l'âge que par le statut financier. L'essentiel de l'action consiste donc à remettre la situation à l'endroit, c'est-à-dire, autant que possible, à revenir à la situation de départ. Mais on ne revient pas si facilement en arrière, comme le découvrent les personnages. La publication de la pièce, qui a connu un succès appréciable à sa création, est suivie d'un entretien entre Justin Laramée, directeur éditorial de

4 Denis Bouchard et Rémy Girard, Tranches de vie, Québec, Éditions Michel Brûlé, 2014, 125 p.

5 Catherine Léger, J'ai perdu mon mari, Montréal, Atelier 10, coll. «Pièces», 2015, 101 p. 
la collection, et Dany Michaud, directeur artistique des Productions À tour de rôle, qui font le point sur l'état actuel du thêâtre d'été, joué souvent en région devant un public de vacanciers, et appellent à la création d'un répertoire neuf et de qualité, qui se démarquerait autant du comique facile que de la folklorisation du «vivre en région", un peu sur le modèle de la pièce de Léger, dont ils célèbrent, de ce point de vue, l'intérêt.

L'introduction d'éléments de satire sociale ajoute du piquant au comique. Dans la préface à En cas de pluie, aucun remboursement ${ }^{6}$, créée à Blainville le 19 juin 2015, dans une mise en scène de l'auteur, Simon Boudreault annonce avoir eu envie de s'amuser (le mot est de lui) avec la politique. S'inspirant de Shakespeare et empruntant une scène de séduction au Cyrano de Bergerac de Rostand, la pièce reste centrée sur les enjeux familiaux, bien que le propos nous entraîne sur le terrain d'un Moyen Âge de pacotille, dans un parc d'attractions pompeusement baptisé «Le Royaume du Super Fun». S'y affrontent le patron (Louis le Juste) et ses capitaines (François le Bel, Henri le Bègue, Lucille la Grasse et Charlotte la Hardie). Or, Louis se fait vieux et il envisage de céder son entreprise à sa fille Marie-Jeanne, personnage peu doué pour les affaires. La guerre éclate entre les sections (la piscine, la restauration, la sécurité, les manèges et jeux d'adresse) pour la place de gérant général. C'est sans compter sur le laissé-pour-compte, le Bossu, qui a un instinct de tueur, et qui n'hésite pas à jouer un double jeu. D'un tableau à l'autre, l'été s'écoule. Louis se désespère des problèmes qui surviennent de manière inhabituelle: le personnel n'entre pas, les animaux quittent leur enclos, les manèges se détraquent. Quand les représentants de la Commission canadienne du tourisme et des loisirs viennent inspecter les cuisines, on comprend que le Bossu, qui a par ailleurs photocopié tous les rapports d'impôt de l'entreprise, a gagné. Il s'adresse au public: «L'histoire est écrite par les vainqueurs. Qu'est-ce que vous croyez? Que vos héros se sont pas battus? » (162) La fin, cependant, reste ouverte, l'auteur n'ayant pas décidé des conditions de la reddition. Peut-être Marie-Jeanne changera-t-elle d'idée. Peut-être Louis fera-t-il face aux autorités...

Il y a des comédies qui sont sages, d'autres qui sont vitrioliques. Révolution à Laval ou Roméo maire, d'après Jarry ${ }^{7}$ de Guillaume Lagarde, créée à l'ESPACE GO le 29 mars 2016, dans une mise en scène de Sébastien Dodge, est de cette sorte. La pièce ouvre sur deux exergues empruntés l'un à Alfred Jarry, «Ceci est sale», et l'autre à Boucar Diouf: «Les politiciens, c'est comme les couches de bébé, il faut les remplacer souvent pour les mêmes raisons!» C'est dire que l'auteur ne fait pas dans la dentelle.

6 Simon Boudreault, En cas de pluie, aucun remboursement, Montréal, Dramaturges éditeurs, 2015, 164 p.

7 Guillaume Lagarde, Révolution à Laval ou Roméo maire, d'après Jarry. Théâtre, Québec, L'instant même, coll. «L'instant scène», 2016, 101 p. 
Dès le début de la pièce, Roméo Urbain est qualifié de «'tit maire de province avec pas d'enverjure!» (12). C'est qu'il est le maire de Mascouche et qu'il rêve de devenir celui de Laval. Peut-être ce rêve est-il surtout celui de sa femme, qui se voit déjà en «Mercédès Urbain. First Lady d'Laval.» (15) La pièce va ainsi de l'inconvenant jusqu'au scatologique, empilant les incidents inavouables. Il faut d'abord remplacer l'échevin Cholette, décédé quand son "pérachute a pas ouvert [le laissant] planté jusqu'au coccux dans l'champ d'pétaques» (28), puis "passer Veilleux à câsserole» (17), au fond d'une cuvette en porcelaine polonaise (Jarry affirmait pourtant que la Pologne n'existait pas) avec la complicité du Chevalier Colon à qui l'on a promis qu'il deviendrait «D. G. d'la Ville de Laval» (18). Une fois élu, Roméo n'est pas au bout de ses peines et il découvre les joies de la mairie d'une grande ville. Promoteurs, hommes d'affaires, mafieux et journalistes véreux se succèdent, emplissant d'enveloppes brunes le sac à main (la sacoche) de Mercédès en échange de quelque milieu humide à asphalter, de quelque dérogation au code du bâtiment ou d'un pourcentage sur les divers contrats de la Ville. La résistance citoyenne s'organise cependant, réunissant «les tout croches, les téteux, les paparasites, les indignes» (87). L'intervention malodorante d'une moufette donnera aux Urbain le temps qu'il faut pour s'enfuir en tandem et se faire discrets jusqu'aux prochaines élections. Alfred Jarry n'aurait pas renié cette pièce, qui emprunte à Ubu roi son caractère inconvenant et sa violence verbale, et qui tire du Hamlet de Shakespeare et de L'aiglon de Rostand quelques scènes et répliques bien sonnantes. En effet, le niveau de langage des personnages est à la hauteur de leur morale, et il faut avoir la langue solidement accrochée pour en apprécier jusqu'au bout l'effet décapant.

À défaut de faire plus vitriolique que Guillaume Lagarde, on peut le faire plus longtemps. Ainsi en est-il de la trilogie que signent Olivier Morin et Guillaume Tremblay et dont les trois volets, intitulés respectivement Clotaire Rapaille ${ }^{8}$, L'assassinat du président ${ }^{9}$ et Épopée nord ${ }^{10}$, ont paru entre 2014 et 2016, mais n'ont encore jamais été présentés ensemble sur la scène. La trilogie est conçue comme un opéra rock, sur une musique dont nous n'avons ni la partition ni les enregistrements, mais qui est signée Navet Confit.

Le premier volet a été créé au Festival OFF de Québec en 2011, avant d'être repris à Montréal, au Zoofest, quelques mois plus tard. On se rappellera Clotaire Rapaille, consultant en marketing, auteur d'une théorie du subconscient collectif des consommateurs qui a fait de lui un expert recherché par plusieurs grandes entreprises et par... la Ville de Québec, qui lui a offert 300000 \$ pour débusquer le «code culturel de la ville». Certains ont vu en lui un charlatan, d'autres en ont fait un poète

8 Olivier Morin et Guillaume Tremblay, Clotaire Rapaille. L'opéra rock. Drame épique et visionnaire, bercé par la douce musique de Navet Confit, Montréal, Les éditions de ta mère, 2014, 137 p.

9 Olivier Morin et Guillaume Tremblay, L'assassinat du président. La première pièce à semer la controverse depuis Le Tartuffe!, Montréal, Les éditions de ta mère, 2015, 179 p.

10 Olivier Morin et Guillaume Tremblay, Épopée nord. Une purge folklorique en soirée canadienne du Thêâtre du Futur, Montréal, Les éditions de ta mère, 2016, 172 p. 
excentrique, cultivant une image de luxe, avec verres fumés et élégante Bentley. Le prologue nous situe dans l'avenir: le pétrole a disparu de la terre, le Québec est devenu indépendant. La vente de l'hydroélectricité a produit une fortune collective et le peuple ne vit que de festival en festival, au cours d'un été qui dure dix mois selon le nouveau calendrier. Du fond de sa prison, où il est écroué pour avoir échoué à identifier le "code culturel du Texas» (11), Rapaille suggère au gouverneur du [sic] Sherbrooke (la ville devenue province se décline désormais au masculin) de construire un «Biodôme de dinosaures mécaniques» (25). Il fera de même avec la province du [sic] Drummondville, qui va se doter d'un réseau de canaux où coulent la sauce brune et le fromage blanc des poutines locales, du [sic] Victoriaville, «où les voitures volent et où chacun a son robot» (42), du Saguenay devenu la province du yéti, alors que Granby pourrait être celle du pain d'épices. Quand Montréal devient à son tour la "City of Colored People and the Circus» (61), les habitants de la ville de Québec, qui attendent toujours leur code culturel, s'insurgent, encouragés par la radio locale (Radio X, évidemment), et ils migrent en masse vers Montréal. La suite de la pièce est à l'avenant, multipliant les incidents et les péripéties quand la situation se dérègle, jusqu'à ce qu'éclate la guerre civile, car il faut vider Montréal des habitants de Québec. Rapaille meurt en héros.

Le deuxième volet, L'assassinat du président, créé dans le cadre du Zoofest à Montréal à l'été 2012 et repris en septembre 2013 au Centre du Thêâtre d'Aujourd'hui, nous ramène un peu en arrière, en 2022. Gilles Duceppe est réfugié en Suisse depuis la défaite du Bloc québécois en mai 2011. François Legault est devenu le premier ministre du Québec et, au référendum qui se tient chaque année, soixante-treize pour cent des citoyens votent pour l'option "peut-être». La moitié nord du Québec a été vendue à la Corée du Sud, le français est optionnel à l'école secondaire, le Parti québécois a disparu de la carte, etc. Au cours d'une entrevue qu'il accorde à la journaliste Anne-Marie Dussault, Duceppe prend conscience du fait qu'il marmonne, lui qui est pourtant fils de comédien, et que, s'il avait parlé plus clairement, l'histoire du Québec aurait pu être changée. Il rentre donc avec précipitation suivre des cours de diction et s'engage dans le débat. Visiblement, ce retour inquiète. Il échappe à un premier attentat à la souffleuse à neige (qui pulvérise le chien de Pauline Marois) et remporte le référendum avec quatre-vingt-sept pour cent des voix, ce qui fait de lui, du même coup, le nouveau président. Il n'échappera cependant pas à un second attentat. Sept ans plus tard, la planète est détruite par une série de catastrophes écologiques, et il ne subsiste désormais que la république des chiens, ce qui nous vaut une sorte d'épilogue intitulé "Legends of the La planète des chiens: Cacahuète (d'après l'œuvre inachevée de Washdi Wouafwaf)", dont on devine les aboutissants.

Dans Épopée nord, créée au Centre du Thêâtre d'Aujourd'hui en janvier 2015, nous sommes en 2035, quelques années après l'indépendance et les cataclysmes écologiques: "D'une christie de province faite en carton, on avait reconstruit un pays en cristal.» (17) Les villes sont à la fine pointe de la technologie, les voitures roulent à l'hydrogène, les maisons ont le chauffage solaire dont les surplus sont redistribués aux sans-abri, les mines du Nord sont nationalisées, le racisme éliminé, les réserves indiennes converties en Centres d'interprétation de la fierté des Premières Nations, très fréquentés par les touristes français, et tout le monde, des plus jeunes 
aux plus vieux, sait turluter et jouer de la cuiller. Or, voici que Fred Pellerin, disparu depuis 2024, apparaît sur l'écran de la télévision. En réalité, ce soir-là, ce sont sept Fred Pellerin qui livrent sur divers médias le message des Premières Nations, "Soyez prêts», avant de se jeter en bas du pont Jacques-Cartier. "Ce jour-là, commente Théophase, on a pas compris grand-chose.» (16) Tout le monde est donc prêt, sans savoir à quoi, jusqu'à ce qu'on découvre que les Amérindiens ont disparu des réserves, au grand dam des touristes, qui demandent à être remboursés. Au bout d'un an, force est de constater que des procédures de clonage ont permis aux Amérindiens d'atteindre une population de huit millions d'habitants. Or, le traité de Valleyfield, signé en 2027, stipule que «le partage du territoire [entre Blancs et autochtones] se fera désormais au prorata du nombre d'habitants» (47). Une fois décantés les territoires protégés puis les terres massacrées par le Plan Nord, et une fois décomptées les maisons individuelles, c'est tout le territoire du sud du Québec qui sera ainsi offert aux virées en quatre-roues et aux activités de chasse et de pêche. Reste donc à contester le statut autochtone des clones.

Rares sont les écrivains qui, au Québec, ont ainsi imaginé l'avenir, ne seraitce que, comme dans le cas présent, sur le mode du comique. Le projet de Morin et de Tremblay ne manque pas d'audace, bien que les trois volets n'aient pas tous été écrits en même temps et que survivent, d'un texte à l'autre, quelques anomalies dans la chronologie, parfois des répétitions incongrues. Il y a un véritable bonheur à envisager ces uchronies sous l'angle des absurdités du présent (si Clotaire Rapaille avait rendu son "code culturel de la ville», si Gilles Duceppe avait moins marmonné) et à soulever du même coup les grands enjeux sociétaux auxquels ce présent devrait rester attentif. En même temps, la trilogie met en scène de nombreuses allusions au théâtre, au cinéma, à la radio et à la chanson. Les tics et les travers du milieu artistique sont à la fois le prétexte de l'invention, ses conditions de possibilités et une grande partie de l'effet comique. La structure des pièces emprunte au répertoire dramatique occidental surtout, encore une fois, à Shakespeare et à Rostand, mais aussi à Molière. Dans le troisième volet de la trilogie, la référence se module de manière plus maîtrisée. La pièce ouvre sur une «Réjouissante entrée du public», dont les accents ne sont pas sans rappeler certaines répliques du Mithridate de Jacques Ferron (Les grands soleils) et qui annonce une assemblée prête à une soirée de contes: «Bon, là, l'histoire qu'on va vous conter, écoutez-la ben comme il faut, parce qu'on la contera pas deux fois.» (7) Dommage! 